\title{
A CONTRIBUTION TO THE THEORY OF SOLUTION.
}

\author{
By E. J. HARTUNG, M.Sc.
}

\author{
(A Papcr read before the Faraday Socicty on Tuesday, May 9, I916, \\ Sir Robert Hadfield, F.R.S., PResident, in the Chair.)
}

When two completely miscible liquids, which do not visibly react with each other, are mixed together in any proportion, the physical properties of the mixture are found in general to differ from those calculated according to the simple mixture law. This divergence has been ascribed to changes in the state of aggregation of the molecules of one or both liquids, to the formation of a definite compound between the two liquids, or to both these causes combined. From a qualitative standpoint the theory is quite satisfactory; on the other hand, it is extremely difficult to apply quantitatively, except in the simplest cases. The reason for this lies in our lack of satisfactory knowledge of the molecular condition of most liquids and of the changes in this condition which take place when different liquids are mixed together. Nevertheless, some fairly satisfactory quantitative results have been obtained in a few simple cases.

Dolezalek * endeavours to apply a quantitative theory in the simplest possible cases-

(i.) Where both liquids are unassociated and one solvate is formed between them.

(ii.) Where one liquid is normal and the other partly associated, while no solvate is formed.

He exemplifies (i.) with mixtures of acetone and chloroform and (ii.) with mixtures of benzene and carbon tetrachloride. By assuming that neither the solvate molecules nor the associated liquid molecules are volatile as such, he shows that a knowledge of the partial pressures of the two components in the vapour phase enables quantitative data as to the extent of solvate formation or association to be determined and also enables the physical properties of any mixture to be calculated. The only physical properties he considers, however, are vapour pressure and density.

In a later paper, Dolezalek and Schulze + make a careful study of the liquid pair ether-chloroform. 'This appears to furnish a simple case of two nonassociated liquids combining to form a single solvate with equimolecular proportions of the parent substances. The authors show how certain physical properties of these mixtures, such as heat capacity, vapour pressure, specific volume refractive index, and viscosity, may be calculated with fair accuracy. Additional evidence of the formation of a solvate is given by the fact that an equimolecular mixture of ether and chloroform solidifies as a single substance when cooled to a temperature about $30^{\circ}$ above the freezing-point of pure ether.

With a view to applying the theory of Dolezalek, Hubbard $\ddagger$ has examined

$$
\text { * Zuit. phy's. Chcm., I908, } 64 . \underset{+}{+} \text { lbid., 1910, 74. } \quad+\text { Ibid., 1913, } 83 .
$$




\section{A CONTRIBUTION TO THE THEORY OF SOLUTION 67}

certain physical properties of a number of binary liquid mixtures. The discussion of his results does not seem to have yet appeared.

Denison,* while recognizing the formation of associated molecules of one or both liquids as a possible cause of departure from the mixture law, mainly devotes his attention to solvate formation, and attempts to show that the divergences are to be ascribed to it, that more than one solvate is not likely to be present, and that the composition of the solvate may be inferred from a quantitative study of such divergences. However, the examples chosen by him to illustrate his theory are not suitable for this purpose.

Holmes $\dagger$ and Holmes and Sageman + consider a number of aqueous and non-aqueous solutions from the property divergence standpoint. They reject the solvate hypothesis and propound a purely physical theory in explanation of their results.

The work in this present paper was undertaken at the suggestion of Professor Orme Masson, after the publication of Denison's results, in order to afford more suitable tests for his theory.

\section{EXPERIMENTAL Work.}

The experimental work in this paper consists of a series of measurements of the density, heat capacity, and heat change on mixing, of mixtures of selected pairs of liquids. The liquids were carefully purified as follows :-

Ethyl Ether.-This was purified in the manner recommended by Wade and Finnemore. $\S$ A considerable quantity of Merck's pure ether was washed in small portions with ten successive changes of distilled water to remove alcohol. The ether was then dried over granular calcium chloride for some days and afterwards over phosphorus pentoxide for a week. The almost anhydrous liquid was then kept in the dark over shredded sodium and sodium amalgam for several months to remove the last traces of water and also any peroxides. When required for use, portions of this stock were distilled from fresh sodium and amalgam and kept in the dark. A density determination gave-

$$
\mathrm{I}_{4}^{20}=0^{\circ} 7 \mathrm{I}^{\circ} 08 .
$$

The density under the same conditions of the ether used by Dolezalek and Schulze \|| was 0.71305 .

Nitrobenzene.-This substance was made by nitrating benzene under the proper conditions, and, after separating and washing the product, fractionally distilling several times until the boiling-point was constant $\left(209^{\circ}\right)$ -

$$
\mathrm{D}_{4}^{20}=\mathrm{I} \cdot 20369 \text {. }
$$

Aniline.-A sample of Schuchardt's pure aniline was repeatedly fractionally distilled through an air condenser until the boiling-point was constant $\left(184^{\circ}\right)$. It was preserved in the dark until required, and was perfectly clear and colourless.

$$
\mathrm{D}_{4}^{20}=\mathrm{I}^{\circ} \mathrm{O} 2 \mathrm{r} 97 . \quad \mathrm{D}_{4}^{25}=\mathrm{I}^{\circ} \text { or } 744 .
$$

Methyl Alcohol.-A A sample of Schuchardt's pure acetone-frez methyl alcohol was boiled for seven hours with metallic calcium under a reflux condenser, closed with a calcium chloride tube, to remove water. It was

* Trans. Faraday Soc., I9I2, VIII, Pt. x.

+ Trants. Chem. Soc., I906, I774. + Ibid., I909, 1919.

\$ Ibid., I909, I842. 
then fractionally distilled from fresh calcium, and the fraction coming over between $65^{\prime} 5^{\circ}$ and $65^{\circ} 24^{\circ}$ (bar. $75^{6} \mathrm{~mm}$., cor.) was collected.

$$
\mathrm{D}_{4}^{25}=0.78740 \text {. }
$$

Carbon Tetrachloride.-A sample of Schuchardt's pure reagent was available, but it proved to contain some considerable amount of carbon disulphide. The method of purification advocated by W. Schmitz-Dumont * was used. This consists in adding to the sample a saturated solution of potassium hydroxide in alcohol and then heating to $60^{\circ}$ for half an hour. If carbon disulphide is present, the liquid turns brown and potassium xanthate is precipitated. The liquid is then washed repeatedly with water until all the alcohol is removed. This process was carried out and, after drying over calcium chloride for some hours, the carbon tetrachloride was fractionated. The product coming over between $76^{\circ} 22^{\circ}$ and $763^{\circ} 8^{\circ}$ (bar. $741 \mathrm{~mm}$., cor.) was collected.

$$
\mathrm{D}_{4}^{25}=\mathrm{I}_{5} \cdot 5469 \text {. }
$$

The density and heat capacity of each pure liquid were then determined at the temperature at which the series of mixtures was to be examined $\left(20^{\circ}\right.$ or $\left.25^{\circ}\right)$. Each mixture of the chosen pair was accurately made up by weighing in a glass-stoppered Erlenmeyer flask, the less volatile liquid being invariably weighed first. The properties of the mixture were then studied.

Density.-This was determined in a narrow-necked density bottle of 25 c.c. capacity, provided with a well-fitting glass stopper. The bottle was carefully calibrated with distilled water beforehand, and was always used with a suitable counterpoise. The measurements were made in a well-regulated water thermostat provided with a standard (Kew certificate) thermometer. The mixture was introduced into the bottle directly from the mixing flask by blowing it through a fine capillary tube. After bringing the level of the liquid in the bottle to a fixed mark on the neck, the inside of the neck above the liquid was carefully dried with narrow strips of filter paper and the stopper inserted, prior to weighing. All density measurements refer to water at $4^{\circ}$ as the standard.

Heat Capacity.-This was determined by a method previously described by the present author. $\dagger$ After the density of the mixture had been determined, the remainder of the liquid in the mixing vessel was introduced by gentle suction into a weight pipette of special form (Fig. I), and from this into the calorimeter, and the heat capacity was determined. The weight pipette consisted of a cylindrical vessel of roo c.c. capacity, provided with a tap (A) and a long, suitably bent delivery tube (B), closed by a well-fitting groundglass cap (C), and carrying a rubber stopper (D) to fit into the opening in the calorimeter.

Heat Change on Mixing.-This was determined by directly mixing the two liquids in known amount in a modified form of the calorimeter used for the heat capacity measurements. The calorimeter (Fig. 2) consisted of an internally silvered thin copper vessel (A) (for one series of mixtures a thin glass calorimeter was used), fixed by means of a rubber ring (C) resting on a cardboard ring (D) in the interior of a silvered Dewar tube (B), which was closed by a well-fitting rubber stopper (E). The temperature was read by a calibrated Beckmann thermometer $(\mathrm{F})$. The liquids were introduced by means of the weight pipette (Fig. I) through a hole in the stopper, ordinarily

* Chent. Zcit., 1897, 2 I.

† Trans. Faraday Soc., 1915, XI, Pt. I. 
closed by a small rubber stopper $(\mathrm{G})$. A thin glass stirring rod $(\mathrm{H})$, terminated by a thin flat disc of glass about $15 \mathrm{~cm}$. in diameter, ensured rapid mixing of the two liquids. This stirring rod was lubricated through the stopper with a little vaseline or phosphoric acid. The under side of the stopper (E) was heavily coated with several layers of tinfoil to protect it from the action of vapours in the calorimeter.

The method of experiment was as follows :-

The two pure liquids were introduced into weight pipettes of similar form (Fig. I) and each carefully weighed. The Dewar tube and calorimeter had been immersed meanwhile in a water thermostat at the temperature of experiment until the reading shown by the Beckmann thermometer was constant (somewhat lower than that shown by the thermostat thermometer). This condition took some considerable time to attain, as the Dewar tube was

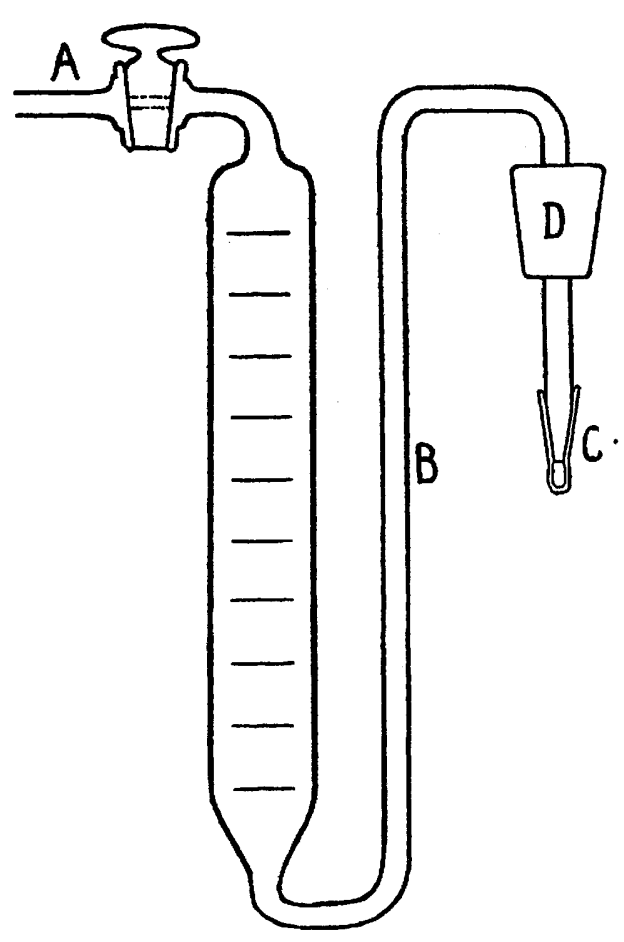

FIG. I.

an efficient insulator. The thermostat was provided with a perforated cardboard cover to diminish radiation. The pipette containing the more volatile liquid was then immersed in the thermostat for half an hour or so with the mouth of the delivery tube over the edge of the bath so that it should not get wet. The glass cap (Fig. I, C) was then removed and the delivery tube inserted into the calorimeter through the hole in the rubber stopper, into which its own stopper fitted. The liquid was introduced by gentle blowing through a calcium chloride tube attached to the other end of the pipette. The calorimeter was then closed and the pipette weighed after again putting on its cap. The pipette containing the second liquid was then immersed in the thermostat, and after half an hour, when the calorimeter temperature was 


\section{A CONTRIBUTION TO THE THEORY OF SOLUTION}

quite constant, the calorimeter was opened, the delivery tube of the pipette inserted as before, and the liquid blown in. The two liquids were rapidly mixed by the stirring rod and the temperature changes noted. Radiation corrections were then applied and the second pipette weighed. The water equivalent of the calorimeter being known, the number of calories evolved or absorbed on mixing was then calculated.

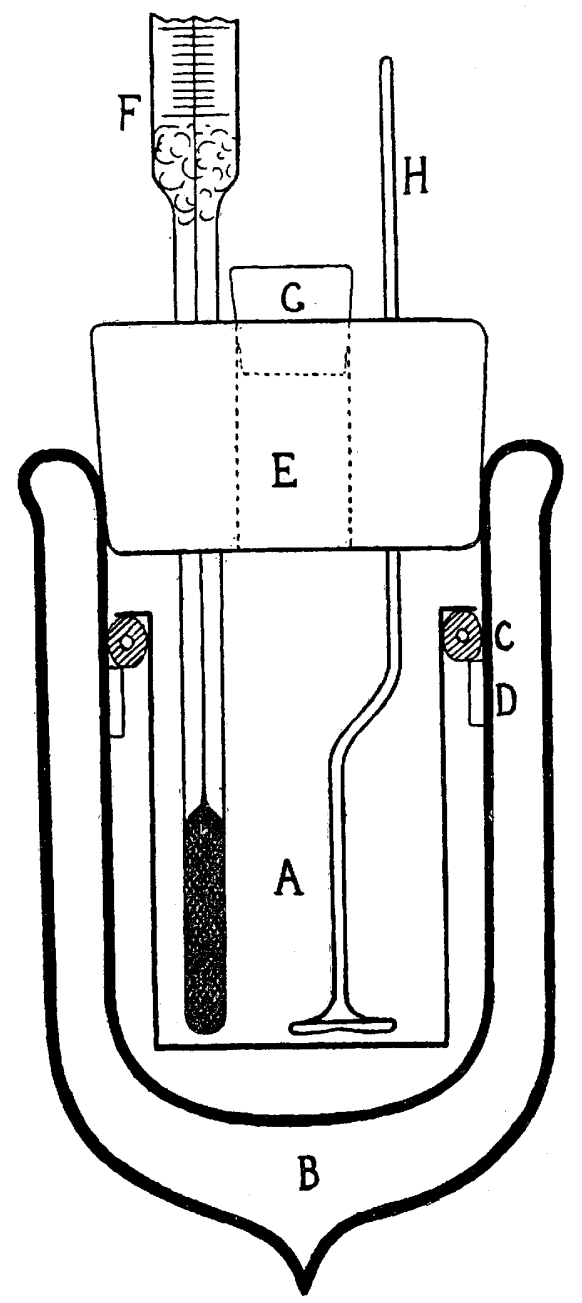

FIG. 2 .

\section{Theoretical Considerations.}

In attempting to establish a theoretical basis on which to consider experimental results one is met at the outset with the difficulty of expressing suitably the concentrations of the various substances present in the chosen mixtures. The molar fraction method recommends itself as being perhaps less open to objection than either the mass or the volume method of calcu- 


\section{A CONTRIBUTION TO THE THEORY OF SOLUTION}

lating concentration; in any case, it is the method which lends itself most simply to mathematical treatment from the mass action standpoint. However, one must remember that the molar fraction method is quite useless in the case of liquids in which the state of molecular aggregation in the liquid state is unknown. This will considerably limit in practice the application of a theory founded on it. In the following discussion the molar fraction method has been adopted.

Let us suppose that we are dealing with two simple unassociated liquids $A$ and $B$, the mixing of which results in the partial formation of a solvate $A_{a} B_{\beta}$. Denison * has shown, in the special case of a solvate $A B_{\beta}$, that the concentration of the solvate is a maximum when the liquid mixture has the synthetic composition denoted by ithe formula of the solvate. The proof can be extended to any solvate $\mathrm{A}_{a} \mathrm{~B}_{\beta}$. Thus we have

$$
\alpha \mathrm{A}+\beta \mathrm{B} \rightleftarrows \mathrm{A}_{a} \mathrm{~B}_{\beta} .
$$

Let $\mathrm{N}$ molecules of $\mathrm{A}$ be mixed with $\mathrm{I}-\mathrm{N}$ molecules of $\mathrm{B}$ with the formation of $x$ molecules of the solvate $\mathrm{A}_{a} \mathrm{~B}_{\beta}$. The total number of molecules present is

$$
\mathrm{I}-(\alpha+\beta-\mathrm{I}) \cdot x \text {. }
$$

Applying mass action considerations, the equilibrium conditions require that

$$
(\mathrm{N}-\alpha x)^{\alpha}(\mathrm{I}-\mathrm{N}-\beta x)^{\beta}=\mathrm{K} x[\mathrm{I}-x(\alpha+\beta-\mathrm{I})]^{\alpha+\beta-\mathrm{I}} .
$$

Differentiating this, we obtain-

$$
(\mathrm{N}-a x)^{\alpha-\mathrm{I}}(\mathrm{I}-\mathrm{N}-\beta x)^{\beta-\mathrm{I}}[\alpha(\mathrm{I}-\mathrm{N})-\beta \mathrm{N}]
$$

$[\mathrm{I}-(\alpha+\beta-\mathrm{I}) x]^{\alpha+\beta-2}[\mathrm{I}-(\alpha+\beta)(\alpha+\beta-\mathrm{I}) x]+(\mathrm{N}-\alpha x) \alpha-\mathrm{I}(\mathrm{I}-\mathrm{N}-\beta x) \beta-\mathrm{I}\left[\alpha^{2}(\mathrm{I}-\mathrm{N}-\beta x)+\beta^{2}(\mathrm{~N}-\alpha x)\right]$.

Hence

$$
\frac{d x}{d \mathrm{~N}}=\text { o when } \frac{\mathrm{N}}{\mathrm{I}-\mathrm{N}}=\frac{\alpha}{\beta} \text {. }
$$

This means that, if we plot the number of molecules of solvate formed per one total synthetic molecule of the mixture against the number of molecules of A present per one total synthetic molecule, we obtain a curve which reaches a maximum at the concentration corresponding to the composition of the solvate.

Further, at one end of the curve (the pure $\mathrm{B}$ end) where $\mathrm{N}=0$ and $x=0$

$$
\frac{d w}{d \mathrm{~N}}=\frac{\mathrm{I}}{\mathrm{K}+\mathrm{I}} \text { if } \alpha=\mathrm{I}
$$

for any other value of $\alpha, \frac{d d x}{d N}=0$.

At the other end of the curve (the pure $\mathrm{A}$ end), $\mathrm{N}=\mathrm{I}$ and $x=0$; therefore

$$
-\frac{d x}{d \mathrm{~N}}=\frac{\mathrm{I}}{\mathrm{K}+\mathrm{I}} \text { if } \beta=\mathrm{I} \text {; }
$$

for any other value of $\beta,-\frac{d x}{d N}=0$.

In words, if the formula of the solvate is the simplest possible, i.e. if $\alpha$ and $\beta$ each equal $\mathrm{I}$, the gradient of the solvate-concentration curve at the pure A

* Loc. cit. 
end must be equal, but of opposite sign, to the gradient at the pure $\mathrm{B}$ end. If $a$ has a value greater than $\mathrm{I}$, then the gradient at the pure $\mathrm{B}$ end is $\mathrm{o}$; similarly for $\beta$ at the pure $A$ end. If $\alpha$ and $\beta$ are each greater than $I$, then the gradients at both ends of the curve equal o.

Now, it is easily shown that the extent of any deviation is directly proportional to the number of solvate molecules formed, or

$$
\Delta=k x,
$$

where $k$ is a constant depending on the molecular values for the particular

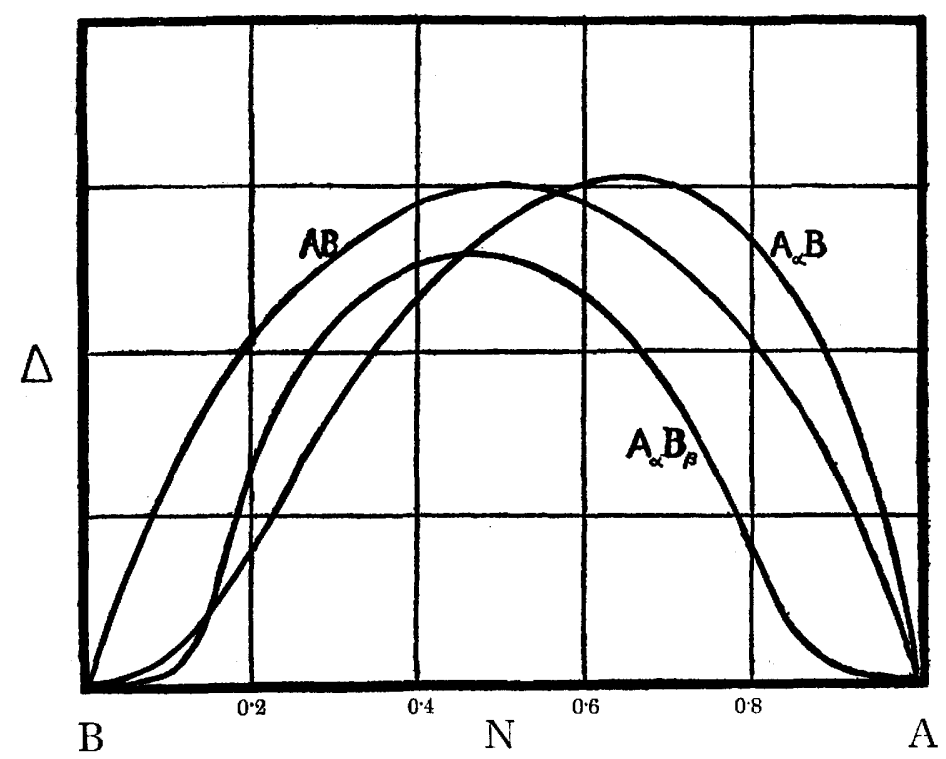

FIG. 3.

property of $A, B$ and $A_{a} B_{\beta}$. Hence the deviation curve also attains its maximum (or minimum in the case of negative deviations) point when

$$
\frac{\mathrm{N}}{\mathrm{I}-\mathrm{N}}=\frac{a}{\beta} \text {, and again } \frac{d \Delta}{d \mathrm{~N}}=0,
$$

at both ends for any values of $\alpha$ and $\beta$ respectively greater than I; but

$$
\frac{d \Delta}{d \bar{N}}=\frac{k}{\mathrm{~K}+1}
$$

at the pure $\mathrm{B}$ end if $\alpha=\mathrm{I}$, and it equals $-\frac{k}{\mathrm{~K}+\mathrm{I}}$ at the pure $\mathrm{A}$ end if $\beta=\mathrm{r}$.

Thus we have three types of curves (Fig. 3), representing the formation of solvates of the composition $\mathrm{AB}, \mathrm{A}_{\alpha} \mathrm{B}$ and $\mathrm{A}_{\alpha} \mathrm{B}_{\beta}$ respectively. Obviously also the deviation curves for all properties of mixtures of a particular pair of liquids must, in general, be similar to each other. 


\section{A CONTRIBUTION TO THE THEORY OF SOLUTION}

For the simple case where $a=\beta=\mathrm{r}$, the equilibrium equation simplifies to-

$$
(\mathrm{N}-x)(\mathrm{I}-\mathrm{N}-x)=\mathrm{K} x(\mathrm{I}-x)
$$

or-

$$
x(\mathrm{I}-x)=\frac{\mathrm{I}}{\mathrm{K}+\mathrm{I}} \cdot \mathrm{N}(\mathrm{I}-\mathrm{N}) .
$$

Since $\Delta=k x$, it follows that-

$$
k=\frac{\Delta}{\mathrm{I}-\frac{k}{\mathrm{~K}+\mathrm{I}} \cdot \frac{\mathrm{N}(\mathrm{I}-\mathrm{N})}{\Delta}}
$$

From this equation we can calculate $k$, the property constant, and $\mathrm{K}$, the equilibrium constant, since values of $\mathrm{N}$ and $\Delta$ may be read from the curve, while $\frac{k}{\mathrm{~K}+\mathrm{I}}$ is the gradient at each end of the curve and can be measured. Or, in order to avoid the inaccuracy of measuring tangents to the curve, we may solve the equation by treating $k$ and $\frac{k}{\mathrm{~K}+\mathrm{I}}$ as two unknown constants and choosing two values of $\mathrm{N}$ on the same property curve. Thus we can evaluate $k$ and $\mathrm{K}$ and, knowing $k$, we can calculate $x$ values from those of $\Delta$ for any mixture and thus plot the true $x$ curve. Further, we can treat different property curves of the same liquid-pair in this way and evaluate the $k$ value for each; and they all should give the same $x$ curve.

It is very doubtful whether any actual cases agree with the simple $A B$ theory, as above, although some may be found to approximate to it closely enough to afford a test. Dolezalek and Schulze* claim that mixtures of ether and chloroform supply such a case, but plotting of their results shows a considerable deviation from perfect symmetry, although this is neglected by them. Notwithstanding Denison's argument to the contrary, it seems probable that, in most cases, two or more solvates are formed, and it becomes very difficult to apply a simple working theory quantitatively. The deviation curves for many pairs of unassociated liquids are quite different for different properties of the same pair, and this makes an explanation on a single solvate basis quite impossible. The assumption of the presence of two or more solvates in such cases gives a satisfactory qualitative explanation at least, for then the deviation curves would be compounded from two or more variables.

A quantitative explanation is not yet possible, because the equations obtained by applying mass action considerations contain several constants for which values cannot be calculated. However, by assigning arbitrary values to these constants it is possible to calculate deviation curves which approximate closely to those obtained by experiment. Further, it is improbable that only one solvate will be present in a given mixture, because the original solvate formed must have some tendency to combine with further molecules of one or both pure components, with the formation of new solvates. Repetitions of this process are possible, but the concentrations of these higher solvates will probably be so small as to be inappreciable.

Throughout the foregoing argument solvate formation alone has been considered and no account has been taken of changes in the state of association of the component liquids on mixing. This limits the testing of the theory in practice to the examination of mixtures of known unassociated liquids, in which mixtures one may reasonably suppose solvate formation to be the predominating factor. In the case of associated liquids it is

* Loc. cit. 


\section{A CONTRIBUTION TO THE THEORY OF SOLUTION}

extremely difficult to develop a satisfactory quantitative theory, owing to the complexity of the phenomena. Dolezalek $*$ has made an attempt to do so in the case of very simple mixtures, but his method can have only a very limited application.

\section{Discussion of the Results.}

For various reasons, the choice of suitable experimental liquids has been somewhat limited. A few words are necessary as to the suitability of the liquids actually chosen for the testing of the solvate theory.

Ether is well known as a typical unassociated liquid.

Aniline and nitrobenzene are found by Dutoit and Mojoiu $\uparrow$ to be slightly associated, while the Ramsay and Shields' method + indicates that both liquids are normal or slightly dissociated. Also Beckmann $\S$ finds that the molecular weight of aniline dissolved in ether is normal.

Methyl alcohol is well known to be strongly associated, the association factor by Ramsay and Shields' method being $3^{\circ} 43$.

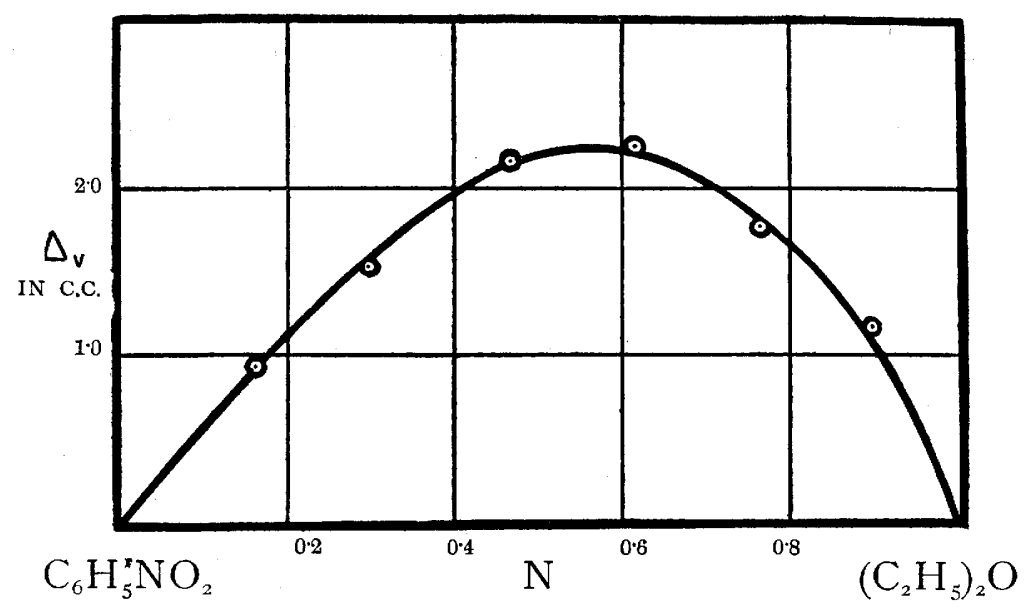

FIG. 4.

Carbon tetrachloride is found to be normal by Dutoit and Mojoiu, $\|$ and this is also indicated by the Ramsay and Shields' method. Dolezalek, on the other hand, calculates a value $I^{\cdot} I_{5}$ at $50^{\circ}$ for the association constant, which fits in with his theory of the conditions obtaining in mixtures of carbon tetrachloride and benzene.

The following symbols are used in the tables of results :-

$M$ the percentage by weight of the more volatile component in the mixture.

$\mathrm{N}$ the number of molecules of this component per one total synthetic molecule of the mixture.

$D$ the density of the mixture at the temperature of experiment, referred to water at $4^{\circ}$.

$\mathrm{V}$ the molecular volume of the mixture in c.c.

$\mathrm{S}$ the specific heat of the mixture.

*. Loc. cit.

Trans. Chcm. Soc., 1893, 63, 1089.

II Loc. cit.

† F. Chim. Phys., I909, 7, I69.

$\$$ Zeit. phys. Chem., 1889, 3, 603 .

I:Zeit. phys. Chem., Igo8, 64 . 
C the molecular heat of the mixture (from a smooth curve).

$\mathrm{E}$ the heat change on mixing per roo gm. of the mixture, in calories.

$\Delta_{v}$ the contraction in volume of the mixture on mixing per one total synthetic molecule, in c.c.

$\Delta_{c}$ the rise in molecular heat on mixing, in calories.

$\Delta_{\mathrm{E}}$ the heat change in calories on mixing per one total molecule of the mixture, positive or negative as indicated.

\section{Nitrobenzene and Ether.}

The experimental results for this liquid-pair are given in the following tables :-

TABle I.

Contractions at $20^{\circ}$.

\begin{tabular}{|c|c|c|c|c|}
\hline M. & N. & D. & V. & $\Delta_{v}$ \\
\hline o & o & $x \cdot 20369$ & $102 \cdot 23$ & o \\
\hline $10 \cdot 35$ & $0 \cdot 1609$ & $I^{\prime} I 3401$ & IOI.55 & 0.93 \\
\hline $20 \cdot 2.1$ & 0.2962 & r.07293 & IOI 17 & I.54 \\
\hline $34 \cdot 34$ & 0.4649 & 0.99462 & 100.83 & $2 \cdot 17$ \\
\hline 48.85 & 0.6134 & 0.92116 & $1000^{\circ} 8$ & $2 \cdot 26$ \\
\hline 65.87 & 0.7623 & 0.84297 & $10 r^{\circ} 69$ & $1 \cdot 78$ \\
\hline $84 \cdot 21$ & 0.8985 & 0.77099 & 102.52 & I.18 \\
\hline 100 & I.00oo & 0.71322 & I03.87 & 0 \\
\hline
\end{tabular}

TABLE II.

Heat Capacity at $20^{\circ}$.

\begin{tabular}{|c|c|c|c|c|}
\hline M. & N. & S. & c. & $\Delta_{c .}$ \\
\hline 0 & o & 0.342 & $42 \cdot \mathrm{I}$ & o \\
\hline IO:35 & $0 \cdot 1609$ & 0.362 & $41 \cdot 7$ & o \\
\hline $20 \cdot 2 \mathrm{I}$ & 0.2962 & 0.382 & $4 x \cdot 5$ & o \\
\hline 34.34 & 0.4649 & 0.408 & $40^{\circ} 9$ & -0.2 \\
\hline $48 \cdot 85$ & 0.6134 & 0.443 & $4 I^{\prime} 2$ & +03 \\
\hline 65.87 & 0.7623 & 0.473 & 40.5 & +0.1 \\
\hline $84.2 \mathrm{I}$ & 0.8985 & 0.512 & $40 \% 5$ & -0.2 \\
\hline 100 & 1.0000 & 0.540 & $40^{\circ} 0$ & 0 \\
\hline
\end{tabular}

The heat change on mixing was found to be very small and no systematic series of measurements of this property was made.

The curve obtained by plotting $\Delta_{v}$ against $\mathrm{N}$ is shown in Fig. 4 . It will be seen that, although the contraction on mixing is considerable, the heat capacity shows practically no deviation from the mixture law. Also, the heat change on mixing is very small. A simple inspection of the contraction curve makes it clear that more than one solvate must be formed in this case, since the curve is not symmetrical and does not reach a maximum at $\mathrm{N}=0^{\circ} 5$ (thus disposing of the simple $\mathrm{AB}$ supposition), and also since the gradients at both ends of the curve have definite values (thus disposing of the $A_{\alpha} B_{\beta}$ supposition). However, the experimental results are too meagre to permit further conclusions to be drawn. 


\section{A CONTRIBUTION TO THE THEORY OF SOLUTION}

Aniline and Ether.

The experimental results for this liquid-pair are given in the following tables :-

TABle III.

Contractions at $20^{\circ}$.

\begin{tabular}{|c|c|c|c|c|}
\hline M. & N. & D. & v. & $\Delta_{v}$ \\
\hline o & o & I.02I97 & 91.07 & o \\
\hline 14.54 & $0.176 \mathrm{I}$ & 0.97322 & 92.19 & $\mathrm{I} \cdot \mathrm{I} 3$ \\
\hline 3078 & $0 \cdot 35^{84}$ & 0.92 IOI & $93 \cdot 66$ & 2.00 \\
\hline $44 * 45$ & 0.5013 & 0.87845 & $95^{\circ}$ II & $2 \cdot 3^{8}$ \\
\hline $54^{\circ} 20$ & o.5979 & 0.84874 & $96 \cdot 28$ & 2.46 \\
\hline 55.85 & 0.6138 & 0.84373 & $96 \cdot 49$ & 2.44 \\
\hline $62 \cdot 19$ & 0.6738 & 0.82466 & 9734 & $2 \cdot 37$ \\
\hline $7 \times \cdot 76$ & $0.76 \div 5$ & o.796́66 & $98 \cdot 75$ & 208 \\
\hline $80 \cdot 38$ & 0.8373 & o:77054 & I00. 55 & $\mathrm{I} \cdot 65$ \\
\hline 100 & $I^{\circ} 0000$ & 0.71308 & 103.90 & o \\
\hline
\end{tabular}

TABLE IV.

Heat Capacity at $20^{\circ}$.

\begin{tabular}{|c|c|c|c|c|}
\hline M. & N. & S. & C. & $\Delta_{c}$ \\
\hline o & o & 0.472 & $43 \cdot 94$ & o \\
\hline $14: 54$ & $0.176 \mathrm{I}$ & 0.490 & $43 \cdot 84$ & 0.59 \\
\hline $30^{\circ} 78$ & $0.35^{84}$ & 0.507 & $43^{\circ} \cdot 7^{2}$ & $\mathrm{r} \cdot \mathrm{I} 8$ \\
\hline $44: 45$ & 0.5013 & 0.520 & $43^{6}$ & $I \cdot 39$ \\
\hline 55.85 & 0.6138 & 0.528 & 42.90 & $I^{\circ} 47$ \\
\hline 62.19 & $0.673^{8}$ & $0: 531$ & $42 \cdot 64$ & $\mathrm{I} \cdot 34$ \\
\hline $71 \cdot 76$ & 0.76 I $_{5}$ & 0.537 & $42 \cdot 15$ & $1 \cdot 20$ \\
\hline $80 \cdot 38$ & 0.8373 & 0.535 & 41.50 & 0.84 \\
\hline 100 & I.0000 & 0.540 & $40 \cdot 02$ & 0 \\
\hline
\end{tabular}

TABLE V.

Heat Change at $20^{\circ}$ (Positive).

\begin{tabular}{|c|c|c|c|}
\hline M. & N. & E. & $\Delta$. \\
\hline $8 \cdot 3^{8}$ & $0 \cdot 103 \mathrm{I}$ & $33 \cdot 82$ & 30.82 \\
\hline $16 \% 2$ & 0.1933 & $54^{\circ} 94$ & $49^{\circ} \mathrm{I} 2$ \\
\hline $25^{\circ} 9^{8}$ & 0.3060 & 92.50 & 80.72 \\
\hline $33 \cdot 10$ & $0 \cdot 3833$ & I $22^{\circ} 98$ & $96^{\circ} 93$ \\
\hline 43.80 & 04947 & $\mathrm{I} 42^{\circ} \mathrm{\circ} 7$ & I 18.88 \\
\hline $54^{\circ} \mathrm{O} 2$ & $0.566 \mathrm{I}$ & I 58.53 & $129^{\circ} 60$ \\
\hline $62 \cdot 88$ & 0.6802 & $160.5 \mathrm{I}$ & 128.64 \\
\hline $75^{\circ 27}$ & 077927 & I $4 I^{\circ} 06$ & I IO० 05 \\
\hline $82^{\circ} 7^{6}$ & 0.8579 & I IOOOI & 84.48 \\
\hline 9130 & 0.9294 & $7 I^{\prime} 3 \mathrm{I}$ & 53.79 \\
\hline
\end{tabular}




\section{A CONTRIBUTION TO THE THEORY OF SOLUTION}

The curves drawn from these three sets of results are given in Figs. 5, 6, and 7 respectively. The departure of each property from the mixture law is strongly marked. The curves of contraction (Fig. 5), of heat capacity deviation (Fig. 6), and of heat evolution (Fig. 7) are similar to each other in general form, but they are not symmetrical and do not reach a maximum at $\mathrm{N}=0^{\circ} 5$, but rather in the neighbourhood of $\mathrm{N}=0^{\circ} 6$. Also, the gradients at the ends of all the curves have definite values. Hence, here again no single solvate supposition will explain the experimental facts, and we are forced to assume the presence of two or more solvates.

Aniline and Methyl Alcohol.

The experimental results for this liquid-pair are given in the following tables :-

TABLE VI.

Contractions at $25^{\circ}$.

\begin{tabular}{|c|c|c|c|c|}
\hline M. & N. & D. & v. & $\Delta_{v}$ \\
\hline $\begin{array}{l}0 \\
8 \cdot 2 \mathrm{I}\end{array}$ & $\begin{array}{l}0 \\
0.2063\end{array}$ & $\begin{array}{l}\text { I.OI 744 } \\
\text { O.99964 }\end{array}$ & $\begin{array}{l}91^{\circ} 47 \\
80^{\circ} 52\end{array}$ & $\begin{array}{l}0 \\
0.49\end{array}$ \\
\hline $14: 22$ & 0.3252 & 0.98602 & $74 \cdot 26$ & 0.70 \\
\hline I9.95 & 0.4199 & $0^{\circ} 9727 \mathrm{I}$ & 69.34 & 0.82 \\
\hline 24.58 & 0.4863 & 0.96193 & $65 \%$ & $0: 87$ \\
\hline $29^{\circ} 40$ & 0.5474 & 0.95056 & 6275 & $0^{\circ} 9^{1}$ \\
\hline $36 \cdot 62$ & 0.6267 & 0.93360 & $5^{8} \cdot 72$ & 0.93 \\
\hline $42^{\circ} 00$ & 0.6777 & 0.92097 & $5^{6 \cdot 13}$ & $0.9 \mathrm{I}$ \\
\hline $54 \cdot 10$ & 0.7740 & 0.89283 & $5+33$ & 0.82 \\
\hline $57.7 \mathrm{I}$ & 0.7986 & $0.884 \mathrm{I} 6$ & $50 \cdot 13$ & 0.78 \\
\hline 7471 & $0.895^{6}$ & 0.84489 & $45^{\circ} 45$ & 0.53 \\
\hline 100 & I.0000 & 0.78740 & $40 \cdot 68$ & 0 \\
\hline
\end{tabular}

Table VII.

Heat Capacity at $25^{\circ}$.

\begin{tabular}{|c|c|c|c|c|}
\hline M. & N. & S. & c. & $\Delta_{\mathrm{e}}$ \\
\hline$\circ$ & & 0.484 & $45^{\circ} 02$ & \\
\hline 8.21 & $0 \cdot 2063$ & 0.512 & $4 r^{\circ} 35$ & $I \cdot 62$ \\
\hline $14 \cdot 22$ & $0.325^{2}$ & $0^{\circ} 534$ & $3^{8 \cdot 95}$ & $2 \cdot 27$ \\
\hline I9.95 & 0.4 I99 & 0.547 & $3^{6.8} 85$ & 2.59 \\
\hline 24.58 & 0.4863 & 0.553 & $35 \times 5$ & $2 \cdot 59$ \\
\hline 2940 & 0.5474 & 0.568 & 3355 & $2 \cdot 56$ \\
\hline $3^{6.62}$ & 0.6267 & $0^{\circ} 57 \mathrm{I}$ & $31 \cdot 35$ & 2.39 \\
\hline $42^{\circ} 00$ & o.6777 & $0^{\circ} 577$ & $30 \% 95$ & $2 \cdot 30$ \\
\hline $54 \cdot$ го & 0.7740 & 0.595 & $27^{\circ} \mathrm{\circ o}$ & $\mathrm{I} \cdot \ddot{8} 2$ \\
\hline $57.7 \mathrm{I}$ & 0.7986 & 0.587 & $26 \cdot 30$ & I 75 \\
\hline $747^{1}$ & 0.8956 & 0.601 & $23 \cdot 15$ & I.09 \\
\hline 100 & I.0OOO & 0.605 & I9.39 & 0 \\
\hline
\end{tabular}


78 A CONTRIBUTION TO THE THEORY OF SOLUTION

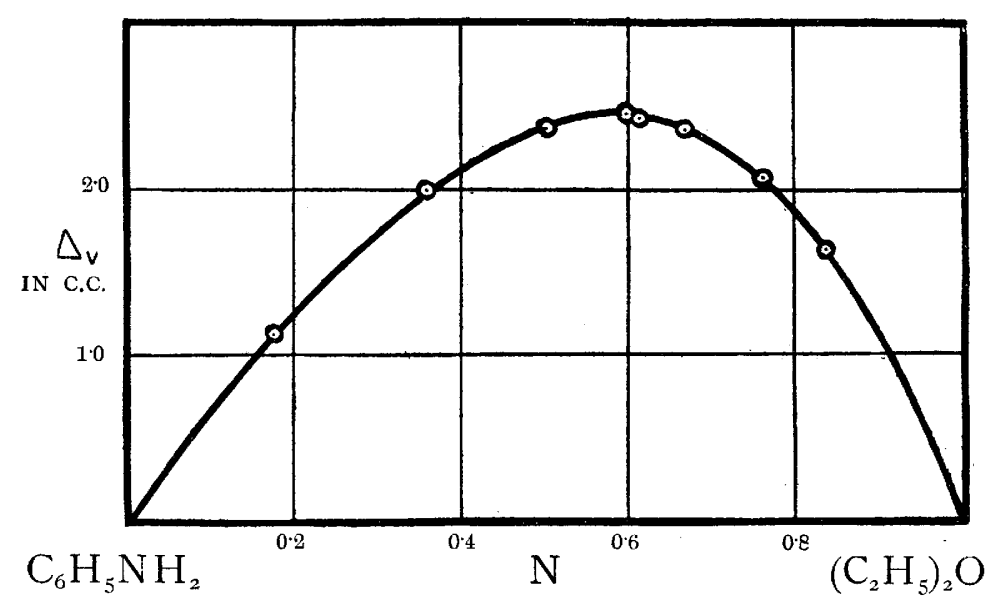

FIG. 5 .

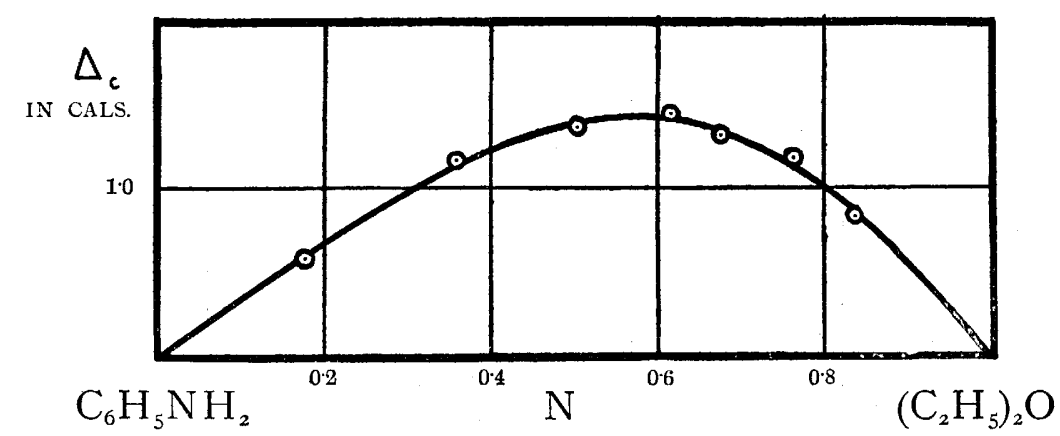

FIG. 6.1

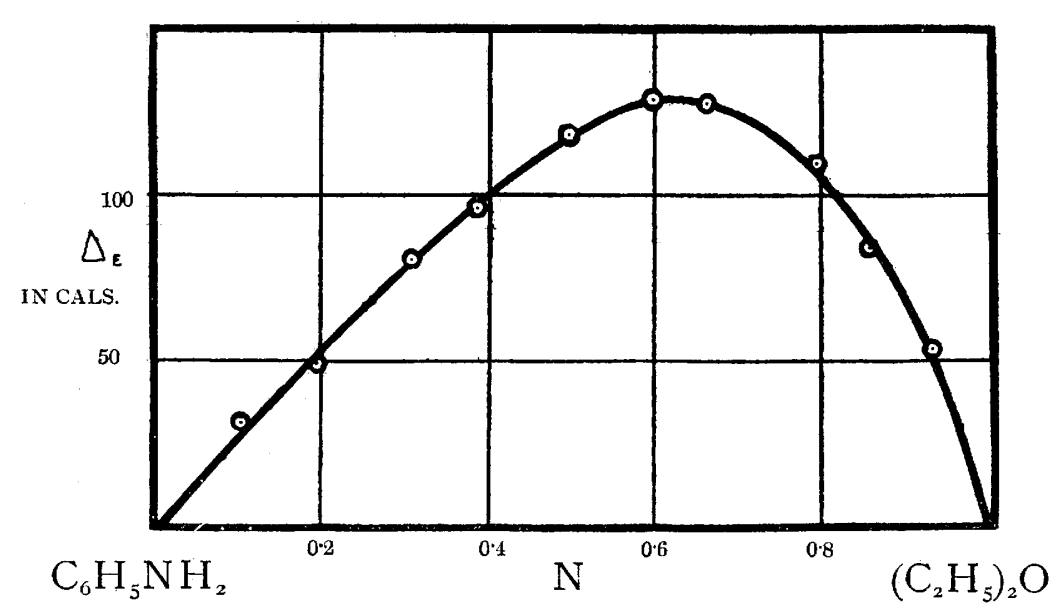

FIG. 7 . 
Table VIII.

Heat Change at $25^{\circ}$.

\begin{tabular}{|c|c|c|c|}
\hline M. & N. & E. & $\Delta_{\mathrm{E}}$ \\
\hline $\begin{array}{r}3 \cdot 12 \\
6 \cdot 32 \\
10 \cdot 58 \\
14 \cdot 48 \\
21 \cdot 01 \\
27 \cdot 65 \\
36 \cdot 01 \\
47 \cdot 30 \\
60 \cdot 61 \\
64 \cdot 32 \\
83.49 \\
86 \cdot 42\end{array}$ & $\begin{array}{l}0.0855 \\
0 \cdot 1639 \\
0 \cdot 2558 \\
0 \cdot 3297 \\
0.4359 \\
0 \cdot 5261 \\
0 \cdot 6205 \\
0 \cdot 7228 \\
0 \cdot 81772 \\
0.8397 \\
0.9362 \\
0.9486\end{array}$ & $\begin{array}{l}-\quad 4.89 \\
-\quad 4.59 \\
+\quad 4.69 \\
+\quad 17.4 \\
+\quad 41.9 \\
+\quad 58.2 \\
+\quad 81.2 \\
+104.9 \\
+ \text { II } 5.1 \\
+114.7 \\
+\quad 80.1 \\
+\quad 63.7\end{array}$ & $\begin{array}{l}-4.30 \\
-\quad 3.81 \\
+\quad 3.63 \\
+12.7 \\
+27.8 \\
+35.5 \\
+44.8 \\
+51.4 \\
+49.7 \\
+47.9 \\
+28.8 \\
+22.4\end{array}$ \\
\hline
\end{tabular}

The curves drawn from these three tables are shown in Figs. 8, 9, and ro respectively. This case is quite unsuitable for consideration from the simple solvate standpoint, since methyl alcohol is notoriously associated. In fact, a solution of aniline in methyl alcohol shows some similarity to one of ammonia in water in that it is capable of dissolving small amounts of copper oxide,

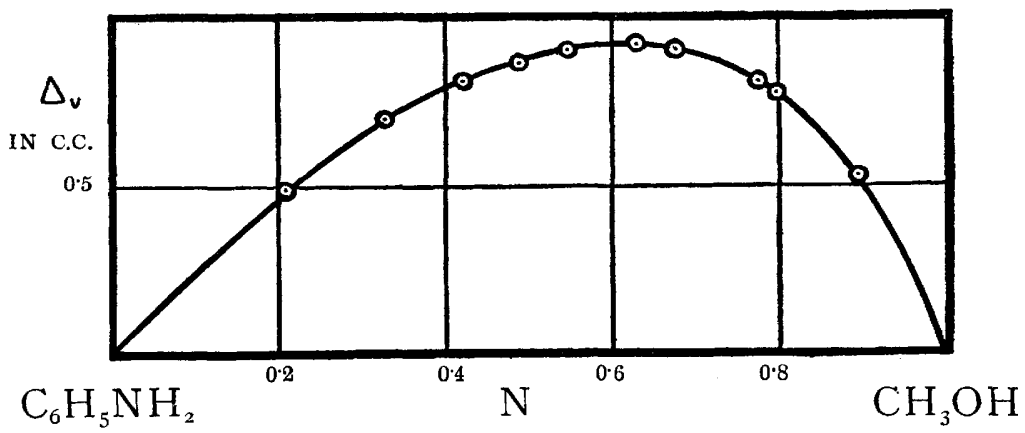

FIG. 8.

giving a green solution. The deviation curves for this mixture are, however, very interesting, although it is quite impossible to fit any simple theory to the experimental results. The contraction curve (Fig. 8) has quite a different maximum to the heat capacity deviation curve (Fig. 9), while the heat change on mixing curve (Fig. 10) is remarkable in showing both a maximum and a minimum. Qualitatively, this behaviour is easily understandable. We may suppose that two simultaneous sets of actions occur on mixing the component liquids-

(i.) Formation of one or more solvates, accompanied by heat evolution.

(ii.) Partial dissociation of the methyl alcohol, accompanied by heat absorption.

When the mixture is rich in alcohol, (i.) predominates and we have a maximum near the alcohol end of the curve. When the mixture is rich in 
aniline, (ii.) predominates and we have a corresponding minimum near the aniline end of the curve. A quantitative theory must be extremely difficult to

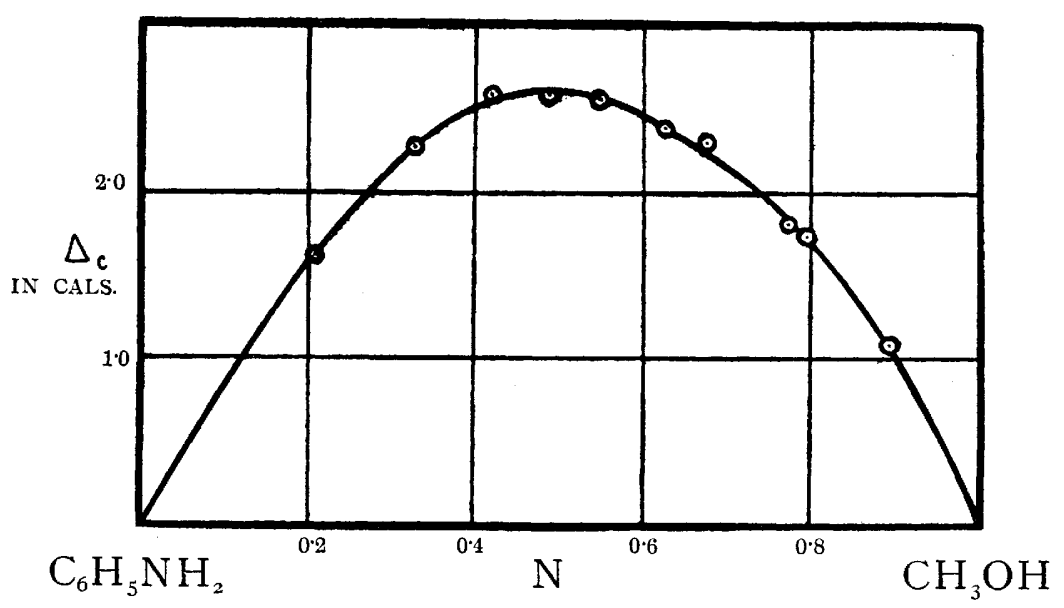

F1G. 9.

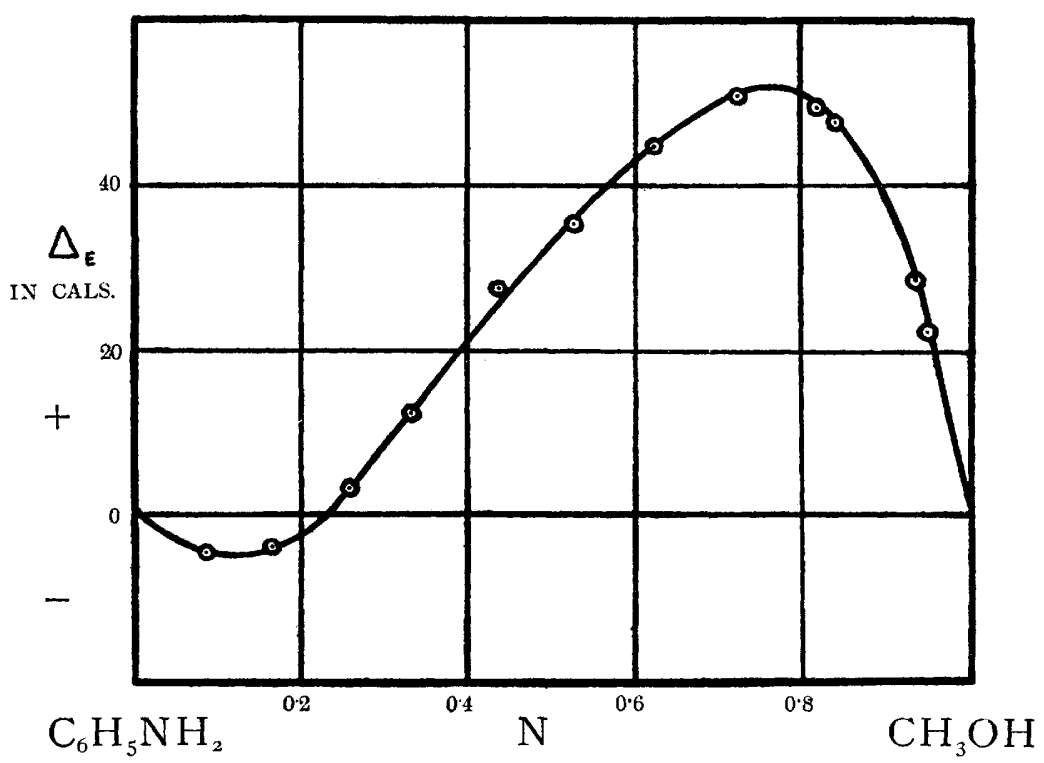

FIG. 10.

apply, in consequence of our lack of knowledge of the association changes which take place when methyl alcohol is mixed with its partner.

\section{Aniline and Carbon Tetrachloride.}

The experimental results for this liquid-pair are given in the following tables :- 
A CONTRIBUTION TO THE THEORY OF SOLUTION 8I

TABle IX.

Contractions at $25^{\circ}$.

\begin{tabular}{|c|c|c|c|c|}
\hline M. & N. & D. & V. & $\Delta_{v_{.}}$ \\
\hline o & 0 & I'OI 748 & $9 \mathrm{I} \cdot 468$ & 0 \\
\hline $154^{\circ}$ & 0.0996 & I.07856 & $9 I \cdot 898$ & O. I 29 \\
\hline $29^{\circ} 60$ & 0.2028 & I. I 4087 & $9^{2} \cdot 382$ & 0.232 \\
\hline $4^{I} \cdot 03$ & 0.2962 & I.19636 & $92 \cdot 843$ & 0.293 \\
\hline $51 \cdot 87$ & 0.3947 & $x \cdot 253^{64}$ & $93 \cdot 372$ & 0.316 \\
\hline 62.08 & 0.4976 & I'3I273 & $93^{\circ} 93^{\circ}$ & 0.330 \\
\hline $7 I \cdot I 7$ & 0.5990 & $I \cdot 36962$ & 94.527 & $0: 303$ \\
\hline $79^{\circ} 06$ & 0.6955 & $\mathrm{I} \cdot 42275$ & $95^{\circ}$ I I & 0.250 \\
\hline 87.05 & 0.8027 & I 48057 & $95^{\circ} 79^{x}$ & 0.167 \\
\hline $93^{\circ} 5^{1}$ & 0.8971 & I.53065 & 96.403 & 0.080 \\
\hline $98 \cdot 14$ & 0.9696 & $I \cdot 56853$ & $96 \cdot 876$ & 0.006 \\
\hline $99: 39$ & 0.9896 & $I^{\prime} 5793^{8}$ & 96.991 & 0.005 \\
\hline 100 & I'OOOO & I.58469 & $97^{\circ} 068$ & o \\
\hline
\end{tabular}

TABLE X.

Heat Capacity at $25^{\circ}$.

\begin{tabular}{|c|c|c|c|c|}
\hline M. & N. & s. & C. & $\Delta_{c .}$ \\
\hline 0 & o & 0.484 & $45^{\circ} \mathrm{O} 2$ & 0 \\
\hline 1546 & o.0996 & 0.446 & $44 \cdot 18$ & 0.52 \\
\hline $29^{\circ} 9^{6}$ & 0.2056 & 0.409 & $43^{\circ} \mathrm{O}_{5}$ & 0.83 \\
\hline $40^{\circ} 47$ & $0^{*} 2915$ & 0.377 & $42 \cdot 05$ & I.OO \\
\hline $5 \times \cdot 87$ & 0.3947 & 0.349 & $40 \cdot 84$ & I. I9 \\
\hline 62.08 & 0.4976 & $0.32 \mathrm{I}$ & 3950 & $\mathrm{I} \cdot 25$ \\
\hline $7 \mathrm{I} \cdot \mathrm{I} 7$ & 0.5990 & 0.295 & 38.20 & 1.33 \\
\hline $79^{\circ} 99$ & 0.7075 & 0.268 & 36.45 & I.06 \\
\hline 87.05 & 0.8027 & 0.244 & 3478 & 0.68 \\
\hline $93 \cdot 85$ & 0.9022 & 0.223 & $33 \cdot 16$ & 0.42 \\
\hline 100 & I.0000 & 0.204 & $3 I^{\circ} 4 I$ & 0 \\
\hline
\end{tabular}

TABLE XI.

Heat Change at $25^{\circ}$ (Negative).

\begin{tabular}{|c|c|c|c|}
\hline M. & N. & E. & $\Delta_{\mathrm{E}}$. \\
\hline $14^{\circ} 67$ & 0.0942 & $08 \cdot 6$ & $97^{\circ}$ \\
\hline $\begin{array}{l}4407 \\
27.26\end{array}$ & $0 \times 1848$ & $162 \cdot 1$ & I69.0 \\
\hline $4 I^{\circ} \cdot 52$ & 03005 & 2127 & $23^{6} 8$ \\
\hline 53.99 & 0.4152 & 238.7 & $282 \cdot 3$ \\
\hline 60.67 & 04827 & 237.8 & $29 \mathrm{I}^{\circ} \mathrm{O}$ \\
\hline $66 \cdot 93$ & 0.5504 & $235^{\circ} \mathrm{I}$ & 2975 \\
\hline 73.08 & 0.6215 & $220^{\circ} 9$ & $288^{\circ} 9$ \\
\hline 8076 & $0.7 \times 75$ & I97.3 & $269^{\circ} 7$ \\
\hline 86.07 & 0.7888 & $174 \cdot 6$ & $246 \cdot 2$ \\
\hline $9 I \cdot 22$ & 0.8627 & $129^{\circ} 2$ & $188^{\circ} 0$ \\
\hline 9431 & 0.9092 & IOO 3 & $148 \cdot 8$ \\
\hline
\end{tabular}


82 A CONTRIBUTION TO THE THEORY OF SOLUTION

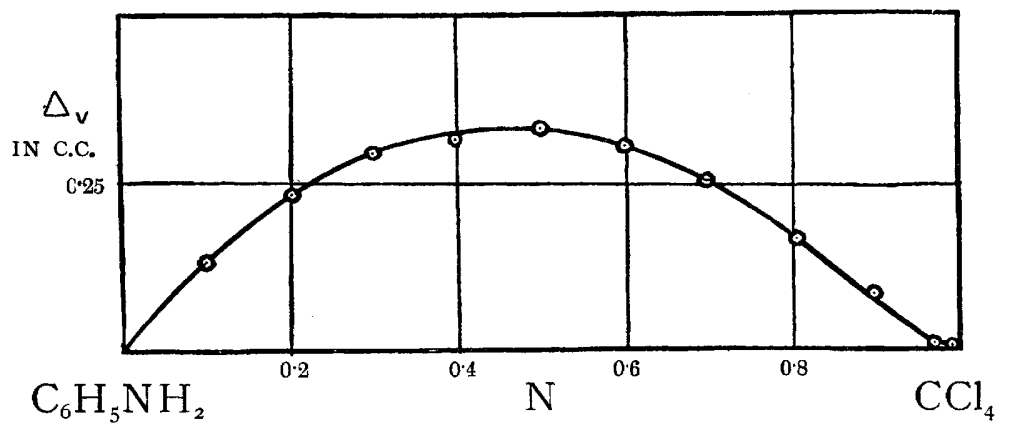

FIG. II.

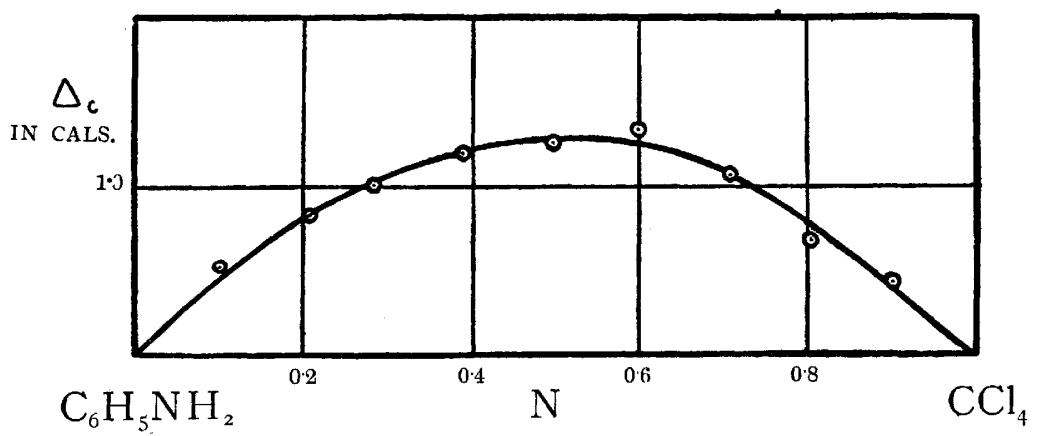

FIG. I2.

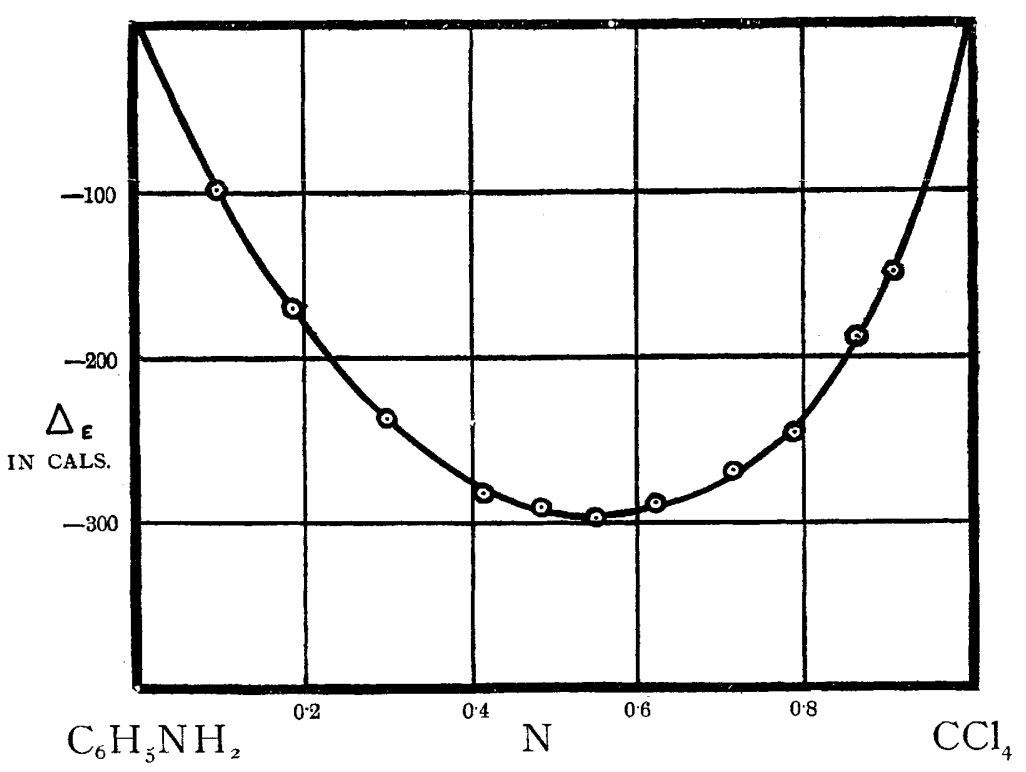

FIG. 13. 


\section{A CONTRIBUTION TO THE THEORY OF SOLUTION 83}

The curves drawn from these three sets of results are given in Figs. II, I 2 and $1_{3}$ respectively. The contraction curve (Fig. II) shows a relatively small volume change, the maximum effect being in the neighbourhood of $\mathrm{N}=0.46$; also, the curve exhibits a point of inflection towards the carbon tetrachloride end and the gradient possibly becomes equal to o at the termination of the curve. The reality of this point of inflection has been carefully verified by repeated experiments. However, the heat capacity deviation curve (Fig. I2) shows no sign of this peculiarity; it also reaches a maximum at $\mathrm{N}=0^{\circ} 5^{2}$. The heat change curve (Fig. $\mathrm{I}_{3}$ ) is similar in these respects, but is remarkable for the very large heat absorption which it exhibits. This is peculiar when contrasted with the small volume contraction shown, instead of a not unreasonably expected expansion.

Again, the single solvate theory is inadequate to explain the results. It is not quite clear why the contraction curve is the only one showing the point of inflection. If it were due to a solvate containing more than one molecule of aniline in its composition, one might expect the deviation curves of other properties to show some evidence of a similar peculiarity. On the other hand, it may be due to slight association of the carbon tetrachloride, which influences the density of the mixtures to a greater extent than it does the heat capacity and the heat absorption on mixing.

\section{Ether and Chloroform.}

This case has been investigated by Dolezalek and Schulze,* and they claim to have shown that the supposition of the presence of a solvate of equimolecular composition explains the experimental results determined for several properties of mixtures of these liquids. However, their curves show considerable deviations from perfect symmetry, which is required by their argument. Their figures for the contractions observed are given in Table XII, their notation having been changed into that adopted in this Paper.

$\mathrm{N}$ represents the synthetic molar fraction of chloroform in the mixture and $\Delta$ the contraction per one total synthetic molecule of the mixture, in c.c.

TABLE XII,

Contractions at $20^{\circ}$.

\begin{tabular}{|c|c|}
\hline N. & $\Delta$. \\
\hline 0.1 & 0.45 \\
\hline 0.2 & 079 \\
\hline 0.3 & 106 \\
\hline 0.4 & 1.27 \\
\hline 0.5 & $r \cdot 30$ \\
\hline 0.6 & I.I6 \\
\hline $\begin{array}{l}0.7 \\
0.8\end{array}$ & $\begin{array}{l}0^{\circ} 97 \\
0^{\circ} 7\end{array}$ \\
\hline $0^{\circ} 9$ & 0.42 \\
\hline
\end{tabular}

The deviation from symmetry is apparent from a simple inspection of these figures; again, if $k$ (the property constant) and $\mathrm{K}$ (the equilibrium constant) are calculated as indicated in the theoretical part of this Paper,

* Loc. cit. 


\section{A CONTRIBUTION TO THE THEORY OF SOLUTION}

different results are obtained according to where the two points on the curve necessary for the calculation are selected.

Thus, using as data

$$
\begin{array}{ll}
\mathrm{N}=0.2 & \Delta=0^{\circ} 79 \\
\mathrm{~N}=0^{\circ} 5 & \Delta=\mathrm{I}^{\circ} 30
\end{array}
$$

we get

$$
k=10.2 \text { and } \mathrm{K}=\mathrm{r} \cdot 24 \text {; }
$$

but if we use as data

$$
\begin{array}{ll}
\mathrm{N}=0.4 & \Delta=\mathrm{I} \cdot 27 \\
\mathrm{~N}=0.7 & \Delta=0.97
\end{array}
$$

we get

$$
k=3.35 \text { and } \mathrm{K}=0.02 \text {. }
$$

The value for $\mathrm{K}$ obtained by Dolezalek and Schultze from vapour pressure considerations is $\mathrm{K}=2 \cdot 96$.

It is evident that this case also cannot be treated from a single solvate standpoint, and we must postulate the existence of two or more solvates.

The evidence adduced in this Paper shows that, in the cases examined, no simple solvate theory will suffice to explain the experimental results, even though the liquids used, with one exception, are little associated. Also, a study of the deviation curves obtained from many other liquid-pairs by various authors leads to the conclusion that only in rare cases, if at all, will the conditions be simple enough to permit of an explanation of the deviations by single solvate formation. In the great majority of cases, the molecular condition of a mixture of two liquids is much more complex, and it becomes very difficult to develop and apply any satisfactory quantitative theory.

The author expresses his sincere thanks to Professor Orme Masson for suggesting the work and for his continual interest and help, especially in the theoretical part, during its performance.

Chemistry Department, Melbourne University, Fanuary i9r6. 